\title{
Maximizing Utilization of Peer Specialists in Community Mental Health: The Next Step in Implementation
}

\author{
Jennifer K. Davis ${ }^{1}$ Sarah I. Pilgrim ${ }^{1}$
}

Received: 2 January 2015/Accepted: 27 March 2015/Published online: 3 April 2015

(C) Springer India Pvt. Ltd. 2015

\begin{abstract}
The successful implementation of peer support services in community mental health centers requires the maximum utilization of peer specialists in order to meet the needs of the consumer, the agency and the peer specialist so that they receive the full benefits of their employment. This study identifies factors that positively and negatively impact the referral to and utilization of peer support services by traditional providers and strategies for achieving optimal utilization.
\end{abstract}

Keywords Peer specialists - Implementation ·

Utilization · Community mental health

\section{Introduction}

The recovery movement in mental health services has produced several innovative practices. Among them is the implementation of peer support services in community mental health centers. The Substance Abuse and Mental Health Services Administration (SAMSHA) identified peer support as one of the ten fundamental components of recovery [1]. The research on the effectiveness of peer provided services has revealed promising but mixed results. Peer supported services have been associated with positive consumer outcomes including reduced symptoms, increased functioning in activities of daily living, enhanced sense of empowerment, and decreased service utilization

Jennifer K. Davis

jenkdavi@ksu.edu

1 Department of Intercampus Academic Programs, Kansas State University-Salina, 2310 Centennial Road, Salina 67401, KS, USA
$[2,3]$. While other studies have found moderate or little impact of peer support services on consumer outcomes [4, 5]. However, the promise of peer provided services supported by the recovery community and research have propelled the implementation of peer support services forward.

In spite of documented support for the utilization of peer support services, implementation of these services has not been without challenges. The research exploring peer support services identifies these implementation challenges as pessimistic attitudes toward recovery among traditional staff, role conflict and confusion among peer providers, and concerns related to professionalism, i.e. confidentiality of client information and consumer/provider boundary issues [6-8]. These studies advise using implementation strategies that focus primarily on integrating peer specialists into the agency workforce. These strategies represent important first steps toward realizing the benefits of peer specialists and their services to the consumer, co-workers and the agency. Although implied by implementation, what have yet to be explicitly addressed in the literature are the factors that impact the utilization of peer specialists by traditional providers.

Peer specialists are trained and certified to provide specific mental health services and serve in several roles including case management $[9,10]$. Peer specialists certification programs exist in 38 of the 52 states, and 8 states currently developing programs. Training programs are not uniform from state to state. The training varies from $40 \mathrm{~h}$ to 16 weeks and most require a written exam for certification [11] While peer specialists establish their own caseload in some treatment settings, typically they depend on the referral of consumers by traditional providers who serve as the primary service providers. Referrals are impacted by several factors including staffing patterns. Peer 
specialists are employed on teams defined as peer support teams or integrated onto traditional treatment teams $[3,12]$. The proximity of peer specialists to the primary provider and ease of referral are factors that may positively or negatively affect referral rates. Without an adequate number of referrals from traditional direct care staff, peer support services may not be offered and used in an optimal way.

The ramifications of inadequate utilization of peer support services for consumers are obvious, as they may not receive services that will be of benefit to them. However, the agency suffers as well, due to loss of potential revenue and lack of adequate billable hours to support the peer specialist positions. The financial consequences to the agency can serve as justification by administrators to curtail the use of peer specialists especially in an agency where the implementation process has been particularly challenging $[8,13]$. The key variable seems to be the traditional direct care provider and her/his decision to utilize peer support services in a consumer's treatment plan.

The successful implementation of peer support services requires the maximum utilization of peer specialists in order to meet the needs of the consumer, the agency and the peer specialist so that they receive the full benefits of their employment. The purpose of this study is to identify factors that positively and negatively impact the referral to and utilization of peer support services by traditional providers and strategies for achieving optimal utilization.

\section{Methodology}

\section{Sample}

A list of 50 community mental health agencies was drawn from the State Community Mental Health Associations in Kansas, Indiana and Ohio. These specific states were selected for their regional geographical locations and their reputation for providing overall support for the utilization of peer provided services.

The community mental health centers located in these states and included in this study met the following criteria: employment of a minimum of 100 direct care staff, provision of comprehensive mental health services including community case management, integrated dual diagnosis treatment, supported employment, outpatient therapy, medication services, and the employment of more than ten peer specialists deployed within departments of peer specialists or integrated into treatment teams. These criteria were selected consistent with what existing research identifies as the various characteristics that may impact the utilization of peer specialists [8, 14]. The staffing pattern of peer specialists served as the distinguishing characteristic of the agencies in this study, to allow for the exploration of the impact of integration of peer specialists on service utilization.

Of 50 community mental health agencies, 19 met the study criteria and three agreed to participate. Each agency was located in a metropolitan area (population 100,000) The agency in Kansas (Agency K) staffed peer specialists on a designated team (Peer Services team with supervisor) and integrated peer specialists onto a team providing Supported Employment (dually supervised by the Team Leader \& Peer Services Team Leader). The agency in Indiana (Agency I) staffed peer specialists on a designated peer specialist team. In each agency, peer specialists were hired through a competitive process to provide services for clients with diagnoses typically found in community mental health centers (i.e. depressive disorders, manic-depression, schizophrenia, personality disorders, substance abuse, etc.). The agency located in Ohio (Agency O) did not employ peer specialists but met all other inclusion criteria and was used for comparison.

\section{Data Collection}

Recruitment of study participants began in February 2012. Potential participants were recruited using flyers describing the study and including contact information for the researcher. The original study design sought to include only direct care workers in its sample, however in the process of recruitment, two supervisors were present in the focus groups unbeknownst to the interviewer. Their input is included in the study database and findings. Participation in the focus groups was voluntary.

Data were collected in five focus groups consisting of case managers, clinicians and some supervisors. Two focus groups were conducted at Agency $\mathrm{K}$, one with providers that worked on teams that utilized peer specialists from a designated department and the second with a team that employed peer specialists as integrated members of their team. The first focus groups consisted of seven direct care providers from case management teams and psychosocial services. The agency tenure of the participants ranged from 1 to 12 years. The second group was attended by seven Employment Specialists from the Supported Employment Team with employment from 3 months to 8 years.

Two focus groups were conducted at Agency I due to the size of the organization and for the convenience of the participants. All participants worked on teams that utilized peer specialists from a designated department. Five participants from case management teams, outpatient therapy, psychosocial services and residential services attended the first focus group, with agency tenure ranging from 6 months to 11 years. The second focus group consisted of ten participants with agency tenure ranging from 7 months to 11 years. 
The final focus group was conducted at Agency $\mathrm{O}$, which did not employ peer specialists. The six participants were employed as case managers on Community Support Services Teams. Lengths of employment at the agency ranged from three to 23 years. The intent of this focus group was to hear from individuals who had no experience working with peer specialists, so that perceptual and attitudinal similarities and differences from others who worked with peer specialists in varying staffing patterns might be identified. During the interview it was revealed that two of the participants had worked with peer specialists in previous employment. This provided the opportunity to make further comparisons.

A semi-structured interview schedule based in Role Theory was constructed to gather data on the following variables: the agency services provided by peer specialists, the impact of peer services on consumer outcomes, the impact of peer specialists on the participants' work with consumers, the impact of peer specialists on the productivity of participants, knowledge of ways that participants access peer services, the different factors that impact the participants' decision to refer a consumer for peer services, and the value that the participants' agencies places on peer services. Additionally, participants were asked for estimates of the percentage of their current caseloads they have referred to peer specialists.

The length of each focus group ranged from 50 to $75 \mathrm{~min}$. Interviews were audio recorded with the permission of each participant. The identity of participants was protected with the use of identification numbers assigned during the interview for use during transcription. Each participant signed an informed consent for participation in the study that included an agreement to keep confidential all information shared during the interview.

\section{Data Analysis}

The data analysis was guided by an inductive approach that identified themes and patterns within and across focus groups [15]. The digital recordings of each focus group were transcribed and entered into a protected computer file. Transcripts were sent to each participant to check for accuracy. Minimal revisions were made to the final transcripts. Two researchers developed a coding scheme for each of the five focus groups and across focus groups. The authors discussed individual coding results and reached consensus on definitions and concepts for 18 codes. The codes were used in further analysis in order to identify emerging codes. None were identified.

The analysis proceeded using microsoft word and track changes to identify themes and sub-themes among the coded data that would allow for a coherent organization of the concepts found in this study. The analysis resulted in the identification of two primary themes, the first being factors that enhance utilization and factors. Within this theme four sub-themes were identified: administrative factors, attitude of the traditional worker, recognition of specific benefits to the consumer, and recognition of benefits to the traditional worker and agency. Codes within each sub-theme were identified from the data. The administrative factors sub-theme included the codes: close proximity to peer specialist, supervisor encouraging utilization and successful integration strategies. Attitude of the traditional worker contained ability to see value in peer services and view of peer specialist as collaborator not competitor as codes. And the codes within the final subtheme, recognition of benefits to the traditional worker and agency included enhancement of assessment information, reinforcing the process and interventions implemented by the worker, increased revenue to the agency and increased consumer satisfaction.

Analysis revealed a second primary theme, which identified barriers to the utilization of peer specialists and their services. Within this theme two sub-themes emerged, functional concerns and attitudinal/relational concerns. Functional concerns consisted of two codes boundary issues and role confusion. The second sub-theme, attitudinal/ relational concerns consisted of the codes attributional stigma and hierarchical stigma. The themes, sub-themes and codes will provide the framework for the presentation of the results.

\section{Results}

\section{Description of Peer Support Services}

Community mental health agencies have the freedom to employ peer specialists to meet service needs resulting in significant variance in the duties of peer specialists, across agencies. In this study it was found that peer specialists provided services to consumers receiving supported employment, integrated dual diagnosis treatment, psychosocial, outpatient, primary care, assertive community treatment, and supervised housing services. In the context of these services, peer specialists facilitated groups, provided skills training, recovery education, community outreach, obtained resources, advocated for consumers, and assisted with crisis case management. Prohibited duties included developing treatment goals and objectives and acting as the primary provider. The primary provider developed the treatment goals and objectives and communicated with the peer specialists regarding the focus of their work with the consumer.

In both agencies employing peer specialists, peer specialists were staffed on specific peer support teams and were accessed through an interdepartmental referral 
process. Peer specialists providing supported employment services at Agency $\mathrm{K}$ were fully integrated onto the team and were assigned a caseload by team members via a shared decision making process.

\section{General Factors that Increase Utilization}

Much of the literature is focused on barriers to the implementation of peer specialists into community mental health agencies. This study resulted in the identification of a number of factors that increase utilization of peer specialists and are found in administrative practices and culture, worker attitudes towards peer specialists, the recognition of positive outcomes that lend validity to peer support services and the recognition of how the worker and agency benefit from the utilization of peer support services.

\section{Administrative Factors}

Having a peer specialist working in close proximity to traditional workers was identified in both agencies (I and $\mathrm{K})$ as being an important precursor to their utilization. The integration of peer specialists onto the treatment team provides direct access to their services. However, regardless of staffing structure, participants reported that having the peer support's office or space in close proximity increased referrals to and use of the peer specialist. A participant from Agency I stated:

If he were in a different building or on a different floor I probably wouldn't have as much opportunity. But it's really easy when his office is close to pop-in and have a quick conversation and discuss possible referrals. (Peer on Designated Team).

However, having the peer specialist integrated onto the team resulted in his/her highest utilization as reported at Agency K:

He is right here, he is in all of our meetings and we discuss the cases with him. More often than not clients are put on his caseload....really the only reason they aren't is if the client refuses the peer services. (Peer Integrated onto Team).

Further evidence of the positive impact of proximity is reflected in the rate of referral to peer specialists. Among participants who did not work in close proximity to a peer specialist (no regular contact or physical availability), between 15 and $28 \%$ of the consumers on their caseload were receiving peer support services. Among participants that worked in close proximity with peer specialists, between 52 and $65 \%$ of their consumers were referred for peer support services. The referral rate on the team with a peer specialist was much higher at $97 \%$ [5].
Agency culture valuing peer specialists emerged as a key factor to the utilization of peer services. Participants from both agencies employing peer specialists expressed that the agency administration places high value on peer support services and most agreed that their supervisors encourage their use. On one team at Agency I, the supervisor was responsible for making referrals so the suggestions for referral were ongoing. On other teams in both agencies it was up to direct care staff to make referrals and all agreed that on occasion supervisors would suggest peer support services for a consumer, resulting in a referral that would not have occurred. One participant stated, "She makes the suggestion and that's probably one of the reasons why we have quite a few of our clients seeing peer specialists...there is a good push and encouragement to utilize the service to the fullest. (Agency I, Peer on Designated Team).

Strategies for successful integration of peer support services in the agency such as thoughtful planning, inclusion of staff in the planning process and development of clear roles for peer specialists effect the immediacy of the utilization of peer specialists and their services. When peer specialists were integrated into the team, this was particularly evident,

We were very careful and thoughtful about how we brought this service onto the team. I was skeptical but I remember changing my mind very quickly. It became apparent to me what a benefit there was, how much they complimented the team, and how really smoothly the whole thing came together. (Agency K, Peer Integrated onto Team).

Thus, it appears taking time for careful planning results in a more immediate willingness to use peer specialists and their services.

\section{Attitude of the Traditional Worker}

The second theme to emerge among the factors that increase peer specialist involvement is the attitude of the traditional worker toward peer specialists and peer support services. Participants that could clearly see the value in peer specialists and their services reported making more frequent referrals. The value of the perspective and experience of the peer specialist was viewed as complementary to the traditional view as opposed to being perceived as a challenge to or less relevant than the traditional perspective of the consumer's experience. This peer perspective is seen as beneficial to both the traditional worker and the consumer. From a participant at Agency K,

We offer important services and support to the client but I think we get stuck because we don't have 
hallucinations, we don't have paranoid thoughts so I don't think that we have that kind of connection with them. The peer supports have had the experience and are really good with the clients and they can make really good progress sometimes. This progress makes me want to use the service more and I have. (Peer on Designated Team).

However, participants remained who continue to struggle with recognizing the value of peer specialists. This will be discussed further in the context of barriers to utilization.

\section{Recognized Benefits to the Consumer}

Human nature compels us to pursue that which will benefit us in matters that are of the most personal importance. The ability to identify the benefits of peer supported services to consumers, workers and the agency proved a particularly important motivator for participants to use peer support services. A type of intuitive cost-benefit analysis seemed to occur in their decision making as they weighed the benefits with the "costs" defined as barriers related to stigma, concerns about lost productivity, and role conflict. The greater the identified benefits and integration of peer specialists, the less concern there was for the challenges to their well-being as employees; challenges that serve as barriers to making referrals to peer specialists.

Positive outcomes for consumers denote one type of benefit that motivates the inclusion of peer services as part of the treatment plan. Participants identified relational factors, skill building and furthering recovery as being of particular importance to the decision to make referrals. Further, peer specialists act as models of recovery and provide consumers with recovery strategies not familiar to traditional workers.

I have had a peer support who has been working with this person who has really bad intrusive thoughts, paranoid thoughts, delusional thinking and she's really clicked with this peer support. She's gotten over this hump from a standstill...he's been able to model for her how he's able to do what he's doing as a peer support with the severe hallucinations he has. This is great for the client and great for me because she is now working with me better than she was. (Agency $\mathrm{K}$, Peer on Designated Team).

This speaks as well to how peer support services reduces the stigma of mental illness for consumers,

Look what this person is able to do, I'm not just my disease. When she feels better about herself we can get a lot more done and she can have a better life. (Agency K, Peer on Designated Team).
Participants were also appreciative of the skill building that occurs through peer support services. This is beneficial to the consumer and also to the worker as they have assistance working with the client to meet these important recovery goals. The skills identified include improved interpersonal skills, coping skills, readiness for independent living and employment and ability to maintain employment. These benefits illustrate the opportunity and merit of a collaborative relationship between traditional workers and peer specialists; each of the providers supporting the other to achieve consumer goals.

The potential of peer specialists to further the recovery of consumers was identified as an additional benefit and motivator for participants. Participants identified increased socialization and symptom management as typical consumer treatment goals. As with skill building, when peer specialists are given the opportunity to collaborate with traditional workers, all benefit from the added support provided to the consumer. As one participant shared,

He was just constantly in his head and wouldn't go out and do different things and just get out of his apartment. There is a fine line between what case management can do and what peer support can do differently just to get him out. The peer specialist was able to get him out more and even got him to his med appointments. We were kind of wary about letting the peer attend the med meetings with him but the client insisted on it and he is now more willing to take his meds. He isn't completely there but he has come a long way. I've been struggling with this case for a very long time. (Agency I, Peer on Designated Team).

\section{Recognized Benefits to the Traditional Worker and Agency}

Achieving consumer goals is of primary importance. However, the benefits of peer support services to workers and the agency contribute further toward assisting consumers in their recovery. The most salient benefit to workers was the opportunity to consult with peer specialists about consumers. Participants from each agency identified this as a real or potential benefit. Interestingly the strength of response regarding this benefit was more significant among participants in agencies with a higher level of integration of peer specialists on the team. Participants not employing peer specialists saw consultation as a possible but not probable occurrence, and participants from the team that have a peer specialist on their team as a member reported consultation as ongoing and of great benefit. Participants at Agency I and Agency $\mathrm{K}$ who worked in close proximity to peer specialists were quite vocal regarding this issue. 
I think the biggest benefit is that I can go to them and collaborate when I have a situation and get a different perspective, which is really helpful. Because a lot of times there will be something that I didn't think of or consider. She has assisted me more than once to better understand and treat my clients. (Agency K, Peer Integrated onto Team).

Other participants who did not have the peer specialist in close proximity reported more reticence regarding the use of peer specialists as consultants. From Agency K, "I'll ask the peer about a client at times and that's okay...but I usually don't have time to track them down...it's just easier to go on." (Peer on Designated Team).Other benefits to the worker included the enhancement of assessment information due to the increased level of trust between the consumer and peer specialist.

I've had good experiences because some of the clients that are guarded with me or the other treaters especially when we first meet them and try to complete the assessment...I've learned things that the client is struggling with through the peer supporter and I use them as much as I can to assist with this. (Agency K, Peer on Designated Team).

Related to this benefit, reinforcing the process and interventions implemented by the worker was identified as an additional benefit, although not in the sense that the peer specialist is acting as an assistant to the traditional worker but as a collaborator in a well-synthesized treatment effort. Only participants from Agency $O$ saw the use of peer specialists as assistants as a potential benefit. This illustrates the consequences of a lack of understanding of the peer specialist role.

In this era of managed care in community mental health, workers face the challenge of providing the best services, in the greatest quantity, using the fewest resources. The phenomenon known as the productivity requirement is a source of concern and anxiety for every participant in this study. Each worker is required to provide a specific number of hours of billable services each week or month. Failure to do so typically results in negative consequences for the employee. This concern yields a hesitance to use peer support services. However, in this study participants who worked in close proximity with peer specialists reported they experienced an increase in their productivity. Participants in focus groups at Agencies I and $\mathrm{K}$ reported similar sentiments, seen in this statement made by a participant from Agency I:

Sometimes by clients will come in for an appointment because they also see him/her on the same day and they get excited about that. So I know that those clients will always come in for their appointments because they see both of us on the same day and that gives them extra motivation to show up for my appointment. (Peer on Designated Team).

A small number of participants still viewed the peer specialist as a competitor for service hours but they did not work on teams in which peer support services were well integrated.

Benefits to workers of using peer support services also translate to benefits to the agency. Participants identified receipt of increased revenue from outside sources such as vocational rehabilitation, increased use of agency services and increased consumer satisfaction with agency services as good outcomes of peer support services. Many funding sources make continued funding conditional on positive outcomes of consumer satisfaction. Services that will increase satisfaction with one service and positively impact satisfaction with other services are quite valuable. As stated by a participant at Agency $\mathrm{K}$,

I have a client that complains about everything and every treater. So we referred him to peer support and he clicked with that guy. It made such a change. He had complained for years and he is complaining much less about all of his services. It's great! (Peer on Designated Team).

Increases in satisfaction can lead to increases in use of agency services, which assist with revenue during a time of shrinking resources.

\section{Barriers to Utilization of Peer Specialist Services}

The findings of this study point to factors that can lead to the increased utilization of peer specialists and their services, which in turn lead to benefits for consumers, peer specialists, traditional workers and mental health agencies. However, there remain well-documented barriers to utilization. These barriers surfaced in this study primarily among those who have little or no exposure to peer specialists, such as those employed by Agency $\mathrm{O}$, and among a few workers whose tenure in the field long predates the recovery movement.

\section{Functional Concerns}

Boundary issues among peer specialists and their clients and traditional co-workers is a well-documented concern among traditional workers. Study participants in each focus group voiced concerns regarding the ability of the peer specialist to transition from friend to provider within the peer group as well as overstepping the scope of their duties. Those working closely with peer specialists pointed out that boundary and role problems were overcome with 
training or through an evaluation process whereby a peer specialist would be identified as being not well suited for the position.

\section{Attitudinal/Relational Concerns}

Participants with the strongest concerns and greatest skepticism regarding the utilization of peer specialists voiced comments reflecting both hierarchical and attributional stigma. Attributional justifications focus on attributes that make a stigmatized person responsible for the stigma. In the context of this study, the attributes of peer specialists that acted as barriers to utilization included concerns about the specialists' decompensation. While not an unrealistic concern, the assumptions about what happens during a period of instability on the part of the peer specialist is based in stigmatizing stereotypes. One participant expressed,

Peers do have their own issues and when a peer decomps it can cause a hassle for the agency because there's people the peer is serving and all of a sudden they're out. And sometimes they can go back on the other side and need support themselves. (Agency I, Peer on Designated Team).

Of course, all human beings have periods of instability and some may have chronic health conditions that require coordination with the agency to deal with their absences. The separation of psychiatric disability as an illness from other illnesses begets a stigmatizing effect that impacts the willingness of the worker to utilize peer specialist services.

Hierarchical justifications are based on the perception of the naturalness and necessity of social hierarchies. The concern regarding professional hierarchy and the place of peer support specialists has been an issue throughout the implementation process. This is manifested in some traditional workers feeling threatened and fearful that peer specialists will replace them. One participant noted.

I've been here a long time and what I do know is that it would seem to me that would be a natural progression to have peer specialists act as primary providers and be a stand alone program. I would be very concerned about that. I think that peer support should be a program in conjunction with professional worker. (Agency K, Peer on Designated Team).

Other doubts included fears of reduced credibility due to peer specialists not always having college level training that might otherwise serve to validate their competence. "I think a lot of the staff members with education have a big struggle with peer providers...we're the ones with the education, we have the degrees." (Agency I, Peer on Designated Team).
The need to discount ability and perspective of peer specialists speaks to hierarchical thinking and the stigmatization of experience and training that leads to tension between non-peer and peer treaters. Missing the point of peer support services all together and causing hesitation to utilize these services.

\section{Discussion}

This study revealed several factors that may positively impact the use of peer specialists and their services. A prominent factor emerging from the data notes the seeming impact of the accessibility of peer specialist on traditional workers who make referrals for their services. The proximity of the peer specialist to the traditional worker clearly increased rates of referral among the study participants. Related to these higher referral rates may be attitudinal differences of traditional providers who have more opportunity for regular contact with peer specialists. Other studies have found that the concerns and stigma regarding peer specialists decrease significantly as traditional workers build working relationships with peer specialists $[10$, 16]. Ready access to the peer specialist creates more opportunities for these relationships to develop. As a result of these findings, agencies may consider how they can creatively use their conceptual and physical space to house peer specialists among traditional workers while maintaining a separate team. Alternatively, agencies may wish to continue to work toward integrating peer specialists onto treatment teams and work through the challenges of doing so as they arise. These findings warrant further exploration of the relationship between proximity and utilization of peer specialists.

A utilization factor that is already well established is the importance of clear roles for peers specialists and HR policies that provide guidance for confidentiality and professional boundaries $[7,16]$. Creating a very specific and clear role for the peer specialist must be accomplished in the context of the team and their services. In addition, including team members and peer specialists in this process is vital [3]. This integration process requires time and investment on the part of all involved, but the benefits to all appear to be well worth the effort.

Accomplishing maximal integration and utilization of peer specialists requires an agency culture that reflects the high value of recovery and peer support services. This culture manifests in a number of dynamics and processes not the least of which is the impact of the communication of this culture by direct supervisors [17]. The findings in this study suggest that the decision to refer a consumer for peer support services depends in part on the encouragement of the direct supervisor to do so. The value and 
commitment of recovery as a cultural element in the agency logically contributes to the supervisor providing this encouragement. The importance of the agency assuring that those who have the most influence on the referral process are truly supportive of peer specialists and their services or utilization will be negatively impacted. Agency administrators might monitor peer specialists' hours of service to identify their underutilization, which might reflect a lack of buy-in by certain supervisors.

Assisting employees to recognize the benefits of using peer support services should have a positive impact on buyin regardless of the position of the traditional employee. There will be peer specialists that do not work out and there will be struggles as roles and boundaries necessarily morph as peer integration evolves. What is important is that the benefits and positive outcomes of peer support services are emphasized on an ongoing basis. This is particularly powerful when coming from direct supervisors and coworkers. When problems occur it is important that employees are reminded of the insidious nature of stigma that might be seen in not fully using methods to provide peer support.

The implementation of peer support services represents an important milestone in the evolution of mental health care. The next step in the process is to ensure that peer specialists are being utilized to the fullest and that the benefits of peer support are realized by the agency as a whole: the consumer, the traditional providers, and the agency administrators. This study reveals that integrating peer specialists into the primary service framework may yield the increased utilization of their services bringing benefit to all who are joined in the helping endeavor, particularly to the peer specialists as they enjoy their status as respected contributors to a professional organization and validation of the value of their experience.

\section{References}

1. Substance Abuse and Mental Health Services Administration (SAMSHA) (2006). SAMSHA issues consensus statement on mental health recovery. http://www.samsha.gov Accessed 25 May 2014.
2. Sledge W, Lawless M, Sells D, Wieland M, O'Connell M, Davidson L. Effectiveness of peer support in reducing readmissions of persons with multiple psychiactric hospitalizations. Psychiatr Serv. 2011;62(5):541-4.

3. Grant E, Reinhart C, Wituk S, Meissen G. An examination of the integration of certified peer specialists into community mental health centers. Community Ment J. 2012;48:477-81.

4. Chinman M, George P, et al. Peer support services for individuals with serious mental illnesses: assessing the evidence. Psychiatr Serv. 2014;65:429-41.

5. Pitt V, Lowe D, Hill S et al. Consumer providers of care for adult clients of statutorymental health services. Cochrane Database of Systematic Reviews 3: CD004807, 2013.

6. Ashcraft L, Anthony W. A story of transformation: an agency fully embraces recovery. Behav Healthc. 2005;17(2):12-21.

7. Gates L, Akabas S. Developing strategies to integrate peer providers in the staff of mental health agencies. Adm Policy Ment Health. 2007;34:293-306.

8. Salzer M, Schwenk E, Brusilovskiy E. Certified peer specialist roles and activities: results from a national survey. Psychiatr Serv. 2010;61(5):520-3.

9. Sells D, Davidson L, Jewell C, Falzer P, Rowe M. The treatment relationship in peer-based and regular case-management for clients with severe mental illness. Psychiatr Serv. 2006;57:1179-84.

10. Rivera J, Sullivan A, Valenti S. Adding consumer-providers to intensive case-management: does it improve outcomes? Psychiatr Serv. 2007;58(6):802-9.

11. Texas Intstitute of Excellence in Mental Health (2014). Peer Specialist Training and Certification Programs: An Overview. http://www.utexas.edu/ssw/cswp/institutes/mentalhealthAccessed 9 Feb 2015.

12. Silver T. Staff in mental health agencies: coping with the dual challenges as providers with psychiatric disabilities. Psychiatr Rehabil J. 2004;28(2):165-71.

13. Salzer M. Certified peer specialists in the United States behavioral health system: an emerging workforce. In: Brown LD, Wituk S, editors. Mental health self-help: consumer and family initiatives. New York: Springer; 2010.

14. Verhaeghe M, Bracke P, Bruynooghie K. Stigmatization and selfesteem of persons in recovery from mental illness: the role of peer support. Int J Soc Psychiatry. 2008;54(3):206-18.

15. Barbour R. Analysing focus groups. In: Fick U, editor. The sage handbook of qualitative data analysis. Sage: Los Angeles; 2013. p. 313-26.

16. Chinman M, Lucksted A, Gresen R, et al. Early experiences of employing consumer-providers in the VA. Psychiatr Serv. 2008;59(11):1315-21.

17. Rapp C, McDiarmid D, Marty D, Ratzlaff S, Collins A, Fukui S. A two-year longitudinal study of the Kansas consumers as providers training program. Psychiatr Rehabil J. 2008;32(1):40-6. 